\title{
Transmission power in some particular cases of bi- or tri-dimensional complex systems
}

\author{
Eustache Mêgnigbêto ${ }^{1,2 *}$ \\ ${ }^{1}$ Bureau of Studies and Research in Information Science, 09 BP 477, Saint Michel, Cotonou, Republic of Benin, ${ }^{2}$ University of Antwerp, \\ Institute for Education and Information Sciences, IBW, Venusstraat 35, B-2000 Antwerp, Belgium
}

\begin{abstract}
In a complex system, mutual information measures the information common to the variables involved. In more than two dimensions, it indicates the level of synergy between variables or the level of self-organization or how central coordinated the system is. Mutual information has a lower and an upper bounds that vary according to the system's configuration. In this paper, we analyse the complex system of the Triple Helix of university-industry-government relationships from the information theory point of view. We find out general characteristics of bi- and tri-dimensional configurations where mutual information reaches its bounds; we compute efficiency, unused capacity and transmission power for such systems as well as for some particular cases.
\end{abstract}

Keywords: Complex systems, information source, mutual information, research collaboration, research policy, transmission power, Triple Helix

\section{INTRODUCTION}

Mutual information was introduced by Leydesdorff $\mathrm{f}^{[1]}$ as an indicator of the Triple Helix of university-industry-government relationships based on Shannon's entropy. ${ }^{[2]}$ Several studies used it to analyse university-industry-government relations in different areas (e.g. $\left.{ }^{[3-7]}\right)$. Mutual information is also called "configurational information" because it results from the system's configuration; it means that it does not depend on one particular variable..$^{[1,4]}$ In more than two dimensions, mutual information is a signed measure: It may be negative, null or positive; ${ }^{[5,8-10]}$ it is not a Shannon-type information. ${ }^{[8-12]}$

According to Leydesdorff, ${ }^{[1]}$ in more than two dimensions,

*Address for correspondence:

E-mail: eustachem@gmail.com

\begin{tabular}{|l|l|}
\hline \multicolumn{2}{|c|}{ Access this article online } \\
\hline Quick Response Code: & \\
\cline { 1 - 2 } & Website: \\
\hline
\end{tabular}

the positivity of the if mutual information indicates a centrally coordinated systemand its negativity the synergy or the level of self-organisation within the system. A null mutual information means independence of variables and is interpreted as absence of interactions between variables; however, Krippendorff ${ }^{11,12]}$ warmed that mutual information could not be used as indicator of unique interactions in complex systems. Mêgnigbêto ${ }^{[13]}$ determined the upper and lower bounds to mutual information in a tri-dimensional system; he demonstrated that they also depend on the system's configuration; then, he proposed efficiency, unused capacity and transmission power. Transmission power measures the efficiency of mutual information; it is the strength of information flow within a system or between its actors and may help in measuring the level of synergy, the pressure or the control exerted within the system.

This paper aims at finding out system configurations that make mutual information reaches its bounds. For such systems, we compute entropies, efficiency, unused capacity and transmission power. We also extend the study to some special cases. The paper is structured as follows: In the next section, we give some methodological background on 
entropy, efficiency, unused capacity, transmission power, and source alphabet; in the third section, we study general characteristics of systems for which mutual information reaches its bound: We compute and discuss these indicators; and, in the last section we compute these indicators in some special cases.

\section{THEORETICAL BACKGROUND}

\section{Entropy of an Event}

Shannon ${ }^{[2]}$ defined the entropy of an event that occurs with the probability $p$ as:

$H=p \times \log _{2} p-(1-p) \times \log _{2}(1-p)$

where $\log _{2}$ is the logarithm to the base 2; the entropy may however be computed to other bases e.g. 3, 4, .., 10). More generally, if $X=\left(x_{1}, x_{2}, \ldots, x_{n}\right)$ is a random variable and its components occur with the probabilities $p_{1}, p_{2}, \ldots$, $p_{n}$ respectively, then the entropy generated by $X$ is ${ }^{[2,14]}$

$H_{X}=-\sum_{i=1}^{n} p_{i} \times \log _{2} p_{i}$

For two random variables $X$ and $Y$ (two dimensions), if $H_{X}$ is the entropy of $X$ and $H_{Y}$ that of $Y$, the joint entropy $H_{X Y}$ of the system of the two variables is equal to the entropy $H_{X}$ plus $H_{Y}$ minus the entropy of the overlay of $X$ and $Y$. The latter is called "rate of transmission" mutual information ${ }^{[1,9,10,15,16]}$ between $X$ and $Y$. The relations between the transmission, $T_{X Y}$, the joint entropy $H_{X Y}$ and the marginal entropies of the variables, $H_{X}$ and $H_{Y}$ are: ${ }^{[2]}$

$H_{X Y}=H_{X}+H_{Y}-T_{X Y}$

and

$T_{X Y}=H_{X}+H_{Y}-H_{X Y}$

In case of three random variables $X, Y$ and $Z$ (three dimensions), the relations between the system's entropy, its transmission, the marginal entropies and the bilateral transmissions are given by (cf..$\left.^{[1,8,17,18]}\right)$ :

$H_{X Y Z}=H_{X}+H_{Y}+H_{Z}-T_{X Y}-T_{X Z}-T_{Y Z}+T_{X Y Z}$

and

$T_{X Y Z}=H_{X}+H_{Y}+H_{Z}-H_{X Y}-H_{Y Z}-H_{X Z}+H_{X Y Z}$

A variable's entropy minus transmission gives its conditional entropy:
$H_{X \mid Y}=H_{X}-T_{Y}$

\section{Efficiency, Unused Capacity and Transmission Power}

A system's entropy reaches its maximum value $H_{\max }$ when its states have the same probability. In the case of the Triple Helix of university-industry-government relationships, if $\mathrm{n}$ is the number of variables within the system, $H_{\max }=\log _{2} M$, where $M=2^{\mathrm{n}}$ or $M=2^{\mathrm{n}}-1 .^{[13]}$ Mêgnigbêto ${ }^{[13]}$ defined the of a system as the fraction of its information production capacity that is really produced; the relative unused capacity is the complement to 1 of the efficiency; the transmission power of a system is the fraction of the maximum value of the transmission devoted to information sharing in the system. It represents the share of the "total configurational information" really produced in the system. In other words, it measures the efficiency of the mutual information. All the three indicators vary from 0 to 1 ; they all are dimensionless and may be expressed as a percentage. ${ }^{[13]}$

In a bi-dimensional system, the transmission power formula is: In a tri-dimensional system, Mêgnigbêto ${ }^{[13]}$ distinguished two types of transmission power: The first one $\left(\tau_{1}\right)$ when the transmission is negative, and the second $\left(\tau_{2}\right)$ when the transmission in positive:

$$
\tau=\left\{\begin{array}{r}
\tau_{1}=\frac{T_{X Y Z}}{H_{X Y Z}-H_{X}-H_{Y}-H_{Z}} \text { if } T_{X Y Z}<0 \\
\tau_{2}=\frac{T_{X Y Z}}{H_{X Y Z}} \text { if } T_{X Y Z}>0 \\
0 \text { if } T_{X Y Z}=0
\end{array}\right.
$$

\section{Information Source, Alphabet and Entropy}

An information source is a random variable that produces symbols. ${ }^{[2,15,16,19]}$ An information source may also be composed of two or more random variables. Assume that the values of the random variable are " 0 , the source does not produce" and " 1 , the source produces". The source's alphabet is then $A=\{0,1\}$. If the source is composed with $\mathrm{n}$ random variables, then the alphabet becomes $A^{n}=\{0,1\}^{n}$; its cardinality is $2^{n}$. Each element of the source's alphabet constitutes an event; each also is a state of the source. ${ }^{[13]}$ For example, the probability distribution of a couple of variable $X$ and $Y$, each with the alphabet $A=\{0,1\}$ is given in Table 1 .

The probability that neither $X$ nor $Y$ produce is $P(X=0$, $Y=0)$; the probability that $X$ does not produce, but $Y$ does is $P(X=0, Y=1)$; the probability that $X$ produces but $Y$ does not is $P(X=1, Y=0)$; the probability that both $X$ and $Y$ produce is $P(X=1, Y=1)$. 
It comes from Table 1 that:

$$
\begin{aligned}
& p(X=0)=p(X=0, Y=0)+p(X=0, Y=1) \\
& p(X=1)=p(X=1, Y=0)+p(X=1, Y=1) \\
& p(Y=0)=p(X=0, Y=0)+p(X=1, Y=0) \\
& p(Y=1)=p(X=0, Y=1)+p(X=1, Y=1) \\
& p(X=0)=p(X=1)=p(Y=0)+p(Y=1)=1 \\
& p(X=0, Y=0)+p(X=0, Y=1)+p(X=1, Y=0) \\
& +p(X=1, Y=1)=1
\end{aligned}
$$

Therefore, entropies follow:

$H_{X}=-p(X=0) \times \log _{2} p(X=0)-p(X=1) \times \log _{2}$ $p(X=1)$

$H_{Y}=-p(Y=0) \times \log _{2} p(Y=0)-p(Y=1) \times \log _{2}$ $p(Y=1)$

$H_{X Y}=-p(X=0, Y=0) \times \log _{2} p(X=0, Y=0)-p(X=0$, $Y=1) \times \log _{2} p(X=0, Y=1)-p(X=1, Y=0) \times \log _{2} p$ $(X=1, Y=0)-p(X=1, Y=1) \times \log _{2} p(X=0, Y=1)$

\section{INDICATORS AT THE BOUNDS TO THE TRANSMISSION IN A BI-DIMENSIONAL SYSTEM}

In a bi-dimensional system, the transmission's lower and upper limits are 0 and $H_{X Y}$, so that $0 \leq T_{X Y} \leq H_{X Y}$

\section{Transmission is Null $\left(T_{X Y}=0\right)$}

The transmission is null if and only if the two variables are independent. ${ }^{[9,10,15]}$ The first example considers two independent and identically distributed variables. Thus, the

\begin{tabular}{|c|c|c|c|}
\hline \multirow[t]{2}{*}{$x$} & \multicolumn{2}{|c|}{$Y$} & \multirow[t]{2}{*}{$p(X)$} \\
\hline & 0 & 1 & \\
\hline 0 & $p(X=0, Y=0)$ & $p(X=0, Y=1)$ & $p(X=0)$ \\
\hline 1 & $p(X=1, Y=0)$ & $p(X=1, Y=1)$ & $p(X=1)$ \\
\hline$p(Y)$ & $p(Y=0)$ & $p(Y=1)$ & 1 \\
\hline
\end{tabular}
four events "Neither $X$ nor $Y$ produce" $(X=0, Y=0)$, “ $X$ doesn't produce but $Y$ does' $(X=0, Y=1)$, “ $X$ produces but $Y$ doesn't $(X=1, Y=0)$ and "Both $X$ and $Y$ produce" ( $X=1, Y=1)$ have the same probability, so that the four atoms generated by the subsets engendered by the random variables have (approximately) the same cardinality. Concretely, it means that in a bi-dimensional system $S=(U, I)$, the events " $U$ only produces" $(U)$, "I only produces" (I), "U and I produce jointly" (UI) and "Neither Unor I produce" $(O)$ have (approximately) the same number of items (e.g.,). Because the alphabet of the source counts four elements $(U, I, U I$ and $O)$, the maximum value of the entropy is $H_{\max }=\log _{2} 4=2$. Therefore, the events are identically distributed, thus, $H_{s}=H_{\mathrm{UI}}=H_{\max }$. Then follow:

$$
\begin{aligned}
& p(U=1)=p(I=1)=p(U=0)=p(I=0)=\frac{1}{2} \\
& H_{U}=H_{1}=-\frac{1}{2} \log _{2} \frac{1}{2}-\frac{1}{2} \log _{2} \frac{1}{2}=-\log _{2} \frac{1}{2}=\log _{2} 2=1
\end{aligned}
$$

$$
H_{U I}=4 \times\left(-\frac{1}{4} \log _{2} \frac{1}{4}\right)=\log _{2} 4=2
$$

We may check that $H_{U I}=H_{U}+H_{I}=1+1=2$ and $T_{U I}=H_{U}+H_{I}-H_{U I}=1+1-2=0$.

The second example relates to university-industry system on the one hand and industry-government system on the other hand drawn from the trilateral university-industry-government relationship in six (They are: Benin, The Gambia, Guinea-Bissau, Liberia, Mali and Niger) West African Member States. ${ }^{[20,21]}$ In these systems, "Other" ('Other' is used by Loet Leydesdorff in a program he coded for mutual information computation (http://www.leydesdorff.net/th2/th4. exe) to designate items not related to university, industry and government) produced, university or government produced, but industry didn't; there isn't any joint output $(U I=I G=0)$; so that $H_{I}=0$, $H_{U I}=H_{U}, H_{I G}=H_{G}$, and $T_{U I}=T_{I G}=0$. The variables are independent; $H_{\max }=\log _{2} 4=2$ bits, transmission power equals zero, relative unused capacity and efficiency vary according to sectorial outputs.

\section{Transmission Equals System's Entropy $\left(T_{X Y}=H_{X Y}\right)$}

The transmission reaches the system's entropy $H_{X Y}$ means that $T_{X Y}=H_{X Y}$

Adding $T_{X Y}-T_{X Y}$ to the right term of Equation (3) leads to

$H_{X Y}=H_{X}+H_{Y}-T_{X Y}+T_{X Y}-T_{X Y}$

and

$H_{X Y}=\left(H_{X}-T_{X Y}\right)+\left(H_{Y}-T_{X Y}\right)+T_{X Y}$ 
Because $H_{X Y}=T_{X Y}$, we can write

$$
\left(H_{X}-T_{X Y}\right)+\left(H_{Y}-T_{X Y}\right)+T_{X Y}=T_{X Y}
$$

which is equivalent to

$\left(H_{X}-T_{X Y}\right)+\left(H Y-T_{X Y}\right)=0$

But $\left(H_{X}-T_{X Y}\right)=H_{X \mid Y}$ and $\left(H_{Y}-T_{X Y}\right)=H_{Y \mid X}$. Besides, $H_{X \mid Y}$ and $H_{Y \mid X}$ are all positive or null; therefore, their sum is null if and only if each of them is. Thus,

$$
\left\{\begin{array}{l}
H_{X \mid Y}=H_{X}-T_{X Y}=0 \\
H_{Y \mid X}=H_{Y}-T_{X Y}=0
\end{array}\right.
$$

The system of equations (23) admits 7 solutions [Appendix 1]. The six West Africa States' university-industry or industry-government systems data; ${ }^{[20,21]}$ and the Korea University-university system in $1999^{[3]}$ are a second illustration $(U=21, I=23, O=0$ and $U I=0 . H_{\mathrm{UI}}=H_{\mathrm{U}}=H_{\mathrm{I}}=T_{\mathrm{UI}}=0.999$ bits, transmission power $=1$ and relative unused capacity $=0.501(M=4)$ or $0.632(M=3)$ illustrate this case. Marginal entropies, system's entropy and transmission are all equal, transmission power is 1 but efficiency, unused capacity; vary depending of variables' value.

\section{INDICATORS AT THE BOUNDS TO THE TRANSMISSION IN A TRI-DIMENSIONAL SYSTEM}

A tri-dimensional transmission has $H_{X Y Z}-H_{X}-H_{Y}-H_{Z}$ as lower bound and $H_{X Y Z}$ as the upper one. ${ }^{[13]}$ The lower bound is negative or null and the upper one positive or null; that reflects the possible negativity of the transmission. The resulting double inequality $H_{X Y Z}-H_{X}-H_{Y}-H_{Z} \leq T_{X Y Z} \leq H_{X Y Z}$ can be split into two others taking into account the transmission's sign. They are: $H_{X Y Z}-H_{X}-H_{Y}-H_{Z} \leq T_{X Y Z} \leq 0$ and $0 \leq T_{X Y Z} \leq H_{X Y Z}$. Therefore, we distinguish below three cases: (i) $T_{X Y Z}=-H_{X}-H_{Y}-H_{Z}$, (ii) $T_{X Y Z}=H_{X Y Z}$ and (iii) $T_{X Y Z}=0$.

Transmission Equals System's Entropy Minus Sum of Sectorial Entropies $\left(T_{X Y Z}=H_{X Y Z}-H_{X}-H_{Y}-H_{Z}\right)$

Equation (5) gives:

$$
\begin{aligned}
& H_{X Y Z}=H_{X}+H_{Y}+H_{Z}-T_{X Y}-T_{X Z}-T_{Y Z}+T_{X Y Z} \text { Thus, } \\
& H_{X Y Z}-H_{X}-H_{Y}-H_{Z}=-T_{X Y}-T_{X Z}-T_{Y Z}+T_{X Y Z}
\end{aligned}
$$

The left term of Equation (15) is the lower bound to $T_{X Y Z}$
If $T_{X Y Z}$ takes this value, then we can write:

$T_{X Y Z}=-T_{X Y}-T_{X Z}-T_{Y Z}+T_{X Y Z}$

Thus,

$-T_{X Y}-T_{X Z}-T_{Y Z}=0$

or $T_{X Y}+T_{X Z}+T_{Y Z}=0$

Knowing that the bilateral transmissions are null or greater than 0 , it comes that $-T_{X Y}-T_{X Z}-T_{Y Z}=0$ if and only if bilateral transmissions $T_{X Y}, T_{X Z}$ and $T_{Y Z}$ are all null, e.g., the random variables $X, Y, Z$ are pairwise independent. Data cubes of Figure 5 and Figure $1 \mathrm{c}$ given by Krippendorff ${ }^{11,12]}$ are some illustrations.

Let us consider a university-industry-government relationships system $S=(U, I, G)$ where individual actors' outputs are null ( $U=I=G=0)$, the joint output UIG also is $(U I G=0)$ but bilateral outputs and 'Other' have (likely) the same value $(U I=U G=I G=\mathrm{O}=\alpha, \alpha>0)$. Let us consider the bilateral system $S=(U, I)$ drawn from this tri-dimensional one. We need first to compute the right output of $U, I$ and $U I$ in the bi-dimensional system following the method given by Mêgnigbêto. ${ }^{[13]}$

$U_{2}=U_{3}+U G_{3}=0+\alpha=\alpha$

$I_{2}=I_{3}+I G_{3}=0+\alpha=\alpha$;

$U I_{2}=U I_{3}+U I G_{3}=\alpha+0=\alpha ;$

$O=\alpha$.

Hence, in the bi-dimensional system $S=(U, I)$, the outputs are $U=I=U I=O=\alpha(\alpha>0)$, which refers to a bi-dimensional system case studied above where $T_{X Y}=0$ and the four events involved are independent and identically distributed. In conclusion, in each bidimensional system derived from the tri-dimensional one, the transmission is null, the system's entropy reaches its maximum value ( 2 bits), and marginal entropies are each equal to 1 bit. Therefore, in the tri-dimensional system, marginal entropies are equal to $1 \mathrm{bit}$, bilateral entropies are equal to 2 bits, bilateral transmissions are null. The considered system is composed with 4 events (UI, UG, $I G$ and $O$ ) that produce each the same number of units. Therefore,

$$
H_{U I G}=\sum_{1}^{4}\left(-\frac{1}{4} \log _{2} \frac{1}{4}\right)=-4 \times \frac{1}{4} \times \log _{2} \frac{1}{4}=\log _{2} 4=2 \text { bits }
$$


By applying the formula in Equation (6), we get $T_{\text {UIG }}=-1$ bit. Data are summarized in Table 2.

Transmission Equals System's Entropy $\left(T_{X Y Z}=H_{X Y Z}\right)$

Let us consider Equation (5):

$H_{X Y Z}=H_{X}+H_{Y}+H_{Z}-T_{X Y}-T_{X Z}-T_{Y Z}+T_{X Y Z}$

If $T_{X Y Z}=H_{X Y Z}$ then it comes that

$H_{X Y Z}=H_{X}+H_{Y}+H_{Z}-T_{X Y}-T_{X Z}-T_{Y Z}+H_{X Y Z}$

That is $H_{X}+H_{Y}+H_{Z}-T_{X Y}-T_{X Z}-T_{Y Z}=0$

Equation (29) may be re-written under two forms:

$\left(H_{X}-T_{X Y}\right)+\left(H_{Y}-T_{Y Z}\right)+\left(H_{Z}-T_{X Z}\right)=0$

or

$\left(H_{X}-T_{X Z}\right)+\left(H_{Y}-T_{X Y}\right)+\left(H_{Z}-T_{Y Z}\right)=0$

With a logical reasoning analogous to that we made in the case of a bi-dimensional system above, we get

$\left\{\begin{array}{l}H_{X \mid Y}=H_{X}-T_{X Y}=0 \\ H_{Y \mid Z}=H_{Y}-T_{Y Z}=0 \\ H_{Z \mid X}=H_{Z}-T_{X Z}=0\end{array}\right.$

or

$\left\{\begin{array}{l}H_{X \mid Z}=H_{X}-T_{X Z}=0 \\ H_{Y \mid X}=H_{Y}-T_{X Y}=0 \\ H_{Z \mid Y}=H_{Z}-T_{Y Z}=0\end{array}\right.$

Equations (32) and (33) are equivalent [Appendix 2].

Equation 32 leads to

$$
\left\{\begin{array}{l}
H_{X}=T_{X Y} \\
H_{Y}=T_{Y Z} \\
H_{Z}=T_{X Z}
\end{array}\right.
$$

The system of equations (34) admits 12 solutions [Appendix 1]: (i) there is no output in the system [Table 3, Row 1], (ii) only one event occurs [Table 3, Rows 2-9], (iii) one variable produce and the other two produce jointly only [Table 3, Rows 10-12]. In the cases where all events' cardinality is null of only one event occurs, the system entropy and transmission, marginal entropies, bilateral entropies, bilateral transmissions are all null; consequently, efficiency is null, unused capacity equals 1 and transmission power is null, by definition. In
Table 2: Indicators of a tri-dimensional system where $\boldsymbol{T}_{\text {UIG }}$ reaches its lower bound

\begin{tabular}{ll}
\hline Indicator & Value \\
\hline Events UI, UG, IG, O & $\alpha>0$ \\
Events U, I, G & 0 \\
$\mathrm{H}_{\mathrm{UIG}}$ & 2 bits \\
Marginal entropies & 1 bit \\
Bilateral entropies & 2 bits \\
Bilateral transmissions & 0 bit \\
Transmission $\left(T_{\mathrm{UIG}}\right)$ & -1 bit \\
Efficiency $(\eta)$ & 0.667 \\
Relative unused capacity $(v)$ & 0.333 \\
Transmission power $\tau=\tau_{1}$ & 1 \\
Observation & $\begin{array}{l}\text { System entropy and transmission, } \\
\text { bilateral entropies and } \\
\text { transmissions, marginal entropies } \\
\text { and transmission, efficiency, relative } \\
\text { unused capacity and transmission } \\
\text { power are invariant whatever } \alpha>0 \text { is }\end{array}$ \\
&
\end{tabular}

Table 3: Solutions of $H_{x \mid y}=0, H_{y \mid z}=0$ and $H_{z \mid x}=0$ (a indicates any positive number except $\mathbf{0}$ ) $(0,0,0)(0,0,1)(0,1,0)(0,1,1)(1,0,0)(1,0,1)(1,1,0)(1,1,1)$

\begin{tabular}{lllllllll}
\hline 1 & 0 & 0 & 0 & 0 & 0 & 0 & 0 & 0 \\
2 & $\alpha$ & 0 & 0 & 0 & 0 & 0 & 0 & 0 \\
3 & 0 & $\alpha$ & 0 & 0 & 0 & 0 & 0 & 0 \\
4 & 0 & 0 & $\alpha$ & 0 & 0 & 0 & 0 & 0 \\
5 & 0 & 0 & 0 & $\alpha$ & 0 & 0 & 0 & 0 \\
6 & 0 & 0 & 0 & 0 & $\alpha$ & 0 & 0 & 0 \\
7 & 0 & 0 & 0 & 0 & 0 & $\alpha$ & 0 & 0 \\
8 & 0 & 0 & 0 & 0 & 0 & 0 & $\alpha$ & 0 \\
9 & 0 & 0 & 0 & 0 & 0 & 0 & 0 & $\alpha$ \\
10 & 0 & 0 & 0 & $\alpha$ & $\alpha$ & 0 & 0 & 0 \\
11 & 0 & 0 & $\alpha$ & 0 & 0 & $\alpha$ & 0 & 0 \\
12 & 0 & $\alpha$ & 0 & 0 & 0 & 0 & $\alpha$ & 0 \\
\hline
\end{tabular}

the case where one sector produces and the two other sectors produce jointly, the system's entropy, the system's transmission, marginal entropies, bilateral entropies, bilateral transmissions are all equal; consequently, transmission power is one. However, unused capacity and efficiency vary according to the variables. Data cubes on Figure 7 given by Krippendorff $f^{12]}$ are an illustrations.

The Transmission is Null $\left(T_{X Y Z}=0\right)$

If the transmission is null, the three variables are mutually independent. The first illustration is the theoretical case where the three variables are independent and identically distributed. Therefore, the eight atoms of the universal set have the same cardinality so that corresponding events have the same probability. Concretely, it means that in a tri-dimensional system $S=(U, I, G)$, the events $U, I, G, U I$, $U G, I G, U I G$ and $O$ produce the same or approximately 
the same number of items (e.g., $p \cong \frac{1}{2}$ ). Therefore, the maximum value of the entropy is $H_{\max } \stackrel{8}{=} H_{U I G}=\log _{2} 8=3$ bits.

$$
\begin{aligned}
H_{U} & =H_{1}=H_{G}=-\frac{1}{2} \log _{2} \frac{1}{2}-\frac{1}{2} \log _{2} \frac{1}{2}=1 \mathrm{bit} \\
H_{U I} & =H_{U G}=H_{I G}=4 \times\left(-\frac{1}{4} \log _{2} \frac{1}{4}\right) \\
& =2 \times \log _{2} 2=2 \mathrm{bits}
\end{aligned}
$$

Bilateral transmission are null; for example $T_{\mathrm{UI}}=H_{\mathrm{U}}+H_{\mathrm{I}}-H_{\mathrm{UI}}=1+1-2=0$. Consequently, $H_{\mathrm{UIG}}=H_{\mathrm{U}}+H_{\mathrm{I}}+H_{\mathrm{G}}=3$ bits. Therefore, efficiency equal 1 , relative unused capacity is null; as transmission is null, transmission power also is.

The second illustration is the example from West African States. ${ }^{[20,21]}$ Six of them have a null industrial output, so $U I, I G$ and $U I G$ are null. $\mathrm{T}_{\mathrm{UIG}}$ is null, but not all bilateral transmissions. Data cube of Figure 6 provided by Krippendorff ${ }^{12]}$ or Figure 1 by Leydesdorff ${ }^{[22]}$ also illustrates this case. These results contrast with the consequence of variables independency theorem stating that when at a system's level the transmission is null, variables are mutually independent and also pairwise independent. ${ }^{[0,10]}$ Accordingly, we should have bilateral transmissions equal 0 ; that is not the case. According to Leydesdorff,, ${ }^{[23]}$ this happens because mutual information in three or more dimensions is not a Shannon-type information. Krippendorff ${ }^{[24]}$ claims that this is a proof that algebra is not able to get unique interactions among three or more variables for the simple reason that probabilities do not take care of circular interactions, which begin with three or more variables. ${ }^{[11,12]}$

\section{OTHER SPECIAL CASES}

Bi-dimensional System $S=(U, I): U=I=U I=\alpha>0$, $O$ is not included

The total output is $3 \alpha$. Therefore, $p(U=1, I=0)=$ $p(U=0, I=1)=p(U=1, I=1)=1 / 3$ and
$H_{U I}=3 \times\left(-\frac{1}{3} \log _{2} \frac{1}{3}\right)=\log _{2} 3=1.585$ bits

$p(U=1)=p(I=1)=1 / 3$ and $p(U=0)=p(I=0)=2 / 3$;

therefore,

$H_{U}=H_{I}=-\frac{1}{3} \log _{2} \frac{1}{3}-\frac{2}{3} \log _{2} \frac{2}{3}=0.918$ bits

And $T_{U I}=H_{I}+H_{U}-H_{U I}=0.252$ bits.

Because $O$ is excluded, $M=2^{\mathrm{n}}-1=3$ and $H_{\max }=\log _{2} 3=H_{\mathrm{UI}}$, so transmission power equals 1 . In this case, marginal entropies, joint entropy, transmission, efficiency, unused capacity and transmission power are invariant whatever $\alpha$ positive [Table 4].

Tri-dimensional System $S=(U, I, G)$. All events have the same probability, $O$ not included:

By considering separately each of the three bilateral systems derived from the trilateral one, $S=(U, I), S=(I, G)$ or $S=(U, G)$ and by proceeding to data correction like in ${ }^{[13]}$ or, ${ }^{[21]}$ we found that marginal entropies are all equal, bilateral entropies the same and bilateral transmissions. Their values are invariant [Table 5].

Tri-dimensional System $S=(U, I, G)$ : All Events have the Same Probability, $O$ not included, $U I G=0$

By considering separately each of the three bilateral systems derived from the trilateral one $S=(U, I), S=(I, G)$ or $S=(U, G)$ and by proceeding to data correction, ${ }^{[13]}$ we compute indicators for such a system [Table 6].

\section{CONCLUSION}

We analysed the complex system of the Triple Helix of university-industry-government relationships from information theory point of view. We found out bi and tri dimensional configurations where mutual information reaches its bounds and computes efficiency, unused capacity and transmission power for such systems as well

\begin{tabular}{|c|c|c|c|c|c|}
\hline Variable & Output & Probability & Entropy (bits) & Indicators & \\
\hline U only & $\alpha$ & 0.333 & $H_{\mathrm{u}}=0.918$ & Transmission $\left(T_{X Y}\right)$ & 0.252 \\
\hline I only & $\alpha$ & 0.333 & $H_{1}=0.918$ & Efficiency ( $\eta$ ) & 1 \\
\hline UI & $\alpha$ & 0.333 & $H_{\mathrm{UI}}=1.585$ & Relative unused capacity (v) & 0 \\
\hline Total & $3 a$ & & & Transmission power $(\tau)$ & 0.159 \\
\hline Observation & \multicolumn{5}{|c|}{$\begin{array}{l}\text { System entropy and transmission, bilateral entropies and transmissions, marginal entropies and transmission, } \\
\text { efficiency, relative unused capacity and transmission power are invariant whatever } \alpha>0 \text { is }\end{array}$} \\
\hline
\end{tabular}
as for some particular cases.

Table 4: Indicators of a bi-dimensional system where $U=I=U I=a>0$ ( 0 not considered) 
Table 5: Indicators of a tri-dimensional system where events are identically distributed ( 0 not included)

\begin{tabular}{|c|c|}
\hline Indicator & Value \\
\hline Events cardinality & $\alpha>0$ \\
\hline$H_{\text {UIG }}$ & 2.807 bits \\
\hline Marginal entropies & 0.985 bits \\
\hline Bilateral entropies & 1.950 bits \\
\hline Bilateral transmissions & 0.020 bits \\
\hline Relative unused capacity $(v)$ & 0.167 \\
\hline Transmission $\left(T_{\mathrm{UIG}}\right)$ & -0.088 bits \\
\hline Efficiency ( $\eta$ & 1 \\
\hline Relative unused capacity $(v)$ & 0 \\
\hline Transmission power $\tau=\tau_{1}$ & 0.591 \\
\hline Observation & $\begin{array}{l}\text { System entropy and transmission, } \\
\text { bilateral entropies and } \\
\text { transmissions, marginal entropies } \\
\text { and transmission, efficiency, relative } \\
\text { unused capacity and transmission } \\
\text { power are invariant whatever } a>0 \text { is }\end{array}$ \\
\hline
\end{tabular}

Table 6: Indicators of a tri-dimensional system where events are identically distributed ( $O$ not included and IUG $=0$ )

\begin{tabular}{ll}
\hline Indicator & Value \\
\hline Events (except UIG) & $\alpha>0$ \\
$H_{\text {UIG }}$ & 2.585 bits \\
Marginal entropies & 1 bit \\
Bilateral entropies & 1.918 bits \\
Bilateral transmissions & 0.082 bits \\
Relative unused capacity $(v)$ & 0.079 \\
Transmission $\left(T_{\text {UIG }}\right)$ & -0.170 bits \\
Efficiency $(\eta)$ & 0.921 \\
Relative unused capacity $(v)$ & 0.079 \\
Transmission power $\tau=\tau_{1}$ & 0.409 \\
Observation & System entropy and transmission, \\
& $\begin{array}{l}\text { bilateral entropies and } \\
\text { transmissions, marginal entropies } \\
\text { and transmission, efficiency, relative } \\
\text { unused capacity and transmission } \\
\text { power are invariant whatever } \alpha>0 \text { is }\end{array}$ \\
\end{tabular}

The bi-dimensional transmission reaches its upper bound, if there is no output within the system, only one actor produces (Other included), or if Other produces and the two actors produce jointly. The tri-dimensional transmission takes its maximum value if there is no output within the system, or if only one actor produce (Other included) or if one actor produces and the other two actors produced jointly. Mutual information in a bi-dimensional system is null if the two variables are independent; in a tri-dimensional system, the mutual information is null is the three variables are mutually independent; however, this doesn't mean that the variables are pairwise independent. In a tri-dimensional system, mutual information reaches its lower bound if variables are pairwise independent. For these cases, transmission power is either null or 1, entropies,
Table 7: Joint probability distribution of $X$ and $Y$

\begin{tabular}{lccc}
\hline $\boldsymbol{X}$ & \multicolumn{2}{c}{$\boldsymbol{Y}$} & $\boldsymbol{p}(\boldsymbol{X})$ \\
\cline { 2 - 3 } & $\mathbf{0}$ & $\mathbf{1}$ & \\
\hline 0 & $p(0,0)$ & $p(0,1)$ & $p(X=0)=p(0,0)+p(0,1)$ \\
1 & $p(1,0)$ & $p(1,1)$ & $p(X=1)=p(1,0)+p(1,1)$ \\
$p(Y)$ & $p(Y=0)=p(0,0)+$ & $p(Y=1)=p(0,1)+$ & 1 \\
& $p(1,0)$ & $p(1,1)$ & \\
\hline
\end{tabular}

Table 8: Conditional probability distribution of $X$ on $Y(X \mid Y)$

\begin{tabular}{ccc}
\hline $\boldsymbol{X}$ & \multicolumn{2}{c}{$\boldsymbol{Y}$} \\
\cline { 2 - 3 } & $\mathbf{0}$ & $\mathbf{1}$ \\
\hline 0 & $\frac{p(0,0)}{p(0,0)+p(1,0)}$ & $\frac{p(0,1)}{p(0,1)+p(1,1)}$ \\
1 & $\frac{p(1,0)}{p(0,0)+p(1,0)}$ & $\frac{p(1,1)}{p(0,1)+p(1,1)}$
\end{tabular}

Table 9: Solutions of equation $H X \mid Y=0$ and $H Y \mid X=0$ (a indicates any positive number except 0 )

\begin{tabular}{ccccc}
\hline & $(\mathbf{0 , 0 )}$ & $\mathbf{( 0 , 1 )}$ & $\mathbf{( 1 , 0 )}$ & $\mathbf{( 1 , 1 )}$ \\
\hline 1 & 0 & 0 & 0 & 0 \\
2 & 0 & 0 & 0 & $\alpha$ \\
3 & 0 & 0 & $\alpha$ & 0 \\
4 & 0 & $\alpha$ & 0 & 0 \\
5 & $\alpha$ & 0 & 0 & 0 \\
6 & 0 & $\alpha$ & $\alpha$ & 0 \\
7 & $\alpha$ & 0 & 0 & $\alpha$ \\
\hline
\end{tabular}

unused capacity or efficiency vary according to the system configuration.

In a bi-dimensional system where Other is not considered, and the two actors produce separately equally as they produce jointly, the system's entropy is invariant, bilateral entropies are invariant and equal, transmission power equal 0.159. In a tri-dimensional system where all events have the same probability and Other not considered, entropies and transmission are invariant, transmission power equals $\tau_{1}=0.591$. In a tri-dimensional system where all events have the same probability, the joint output of the three actors is null and Other not considered, entropies and transmission are invariant, and transmission power equals $\tau_{1}=1$.

In the reflexions above, we made the equivalence between "events have the same probability" and the "associated sets have the same cardinality". In fact, the associated set to events may have likely the same cardinality; the result is approximately identic. For example, in case of a bi-dimensional system, if the four cardinalities are 100, 98, 96 , and 94 yields the same indicators as all four cardinalities are equal to 100. 


\section{REFERENCES}

1. Leydesdorff L. The mutual information of university-industry-government relations: An indicator of the Triple Helix dynamics. Scientometrics 2003;58:445-67.

2. Shannon CE. A mathematical theory of communication. Bell Syst Tech J 1948;27:379-423, 623-56.

3. Khan FG, Park HW. Measuring the Triple Helix on the web: Longitudinal trends in the university-industry-government relationship in Korea. J Am Soc Inf Sci 2011;62:2443-55.

4. Leydesdorff L, Sun Y. National and international dimensions of the Triple Helix in Japan: University-industry-government versus international co-Authorship relations. J Am Soc Inf Sci 2009;60:778-88.

5. Leydesdorff L. Configurational information as potentially negative entropy: The Triple Helix model. Entropy 2008;10:391-410.

6. Mêgnigbêto E. Triple Helix of university-industry-government relationships in West Africa. J Scientometric Res 2014;2:54-62.

7. Ye YF, Yu SS, Leydesdorff L. The Triple Helix of university-industry-government relations at the country level, and its dynamic evolution under the pressures of globalization. J Am Soc Inf Sci Technol 2013;64:2317-25.

8. Leydesdorff L, Ivanova IA Mutual redundancies in interhuman communication systems: Steps towards a calculus of processing meaning. J Assoc Inf Sci Technol 2014;65:386-99.

9. Yeung RW. A First Course in Information Theory. Boston: Kluwer Academic Publishers; 2001.

10. Yeung RW. Information Theory and Network Coding. New York: Springer; 2008.

11. Krippendorff K. Information of interactions in complex systems. Int J Gen Syst 2009;38:669-80.

12. Krippendorff K. Ross Ashby's information theory: A bit of history, some solutions to problems, and what we face today. Int J Gen Syst 2009;38:189-212.

\section{APPENDIX 1: CONDITIONS FOR THE TWO (THREE) BI (TRI)-DIMENSIONAL CONDITIONAL ENTROPIES ARE NULL}

Let us consider two random variables $X$ and $Y$ each with the alphabet $A=\{0,1\}$. The joint probability distribution of $X$ and $Y$ and the conditional probability distribution are given in Table 7 and Table 8 respectively.

By definition, the conditional entropy $H_{X \mid Y}$ is ${ }^{[9,10]}$ :

$$
\begin{aligned}
H_{X Y Y} & =\sum_{x, y} p(x, y) \times \log _{2} p(x \mid y) \\
& =-\sum_{y} p(y) \times H_{X Y Y=y}
\end{aligned}
$$

Then, from Table 1, we can write

$$
\begin{aligned}
& H_{X \mid Y} \\
& \quad=-\frac{p(0,0)}{p(0,0)+p(1,0)} \log _{2} \frac{p(0,0)}{p(0,0)+p(1,0)} \times(p(0,0)+p(1,0))
\end{aligned}
$$

13. Mêgnigbêto E. Efficiency, unused capacity and transmission power as indicators of the Triple Helix of university-industry-government relationships. J Informetr 2014;8:284-94.

14. Shannon CE, Weaver W. The Mathematical Theory of Communication. Urbana: University of Illinois; 1949.

15. Cover TM, Thomas JA. Elements of Information Theory. $2^{\text {nd }}$ ed. Hoboken: J. Wiley; 2006.

16. Mori Y.Electronics for signal processing. Information and coding theory: Analogic signal, electronic signal and application in telecommunications. Paris: Lavoisier; 2006.

17. Abramson N. Information Theory and Coding. New York, etc: McGraw-Hill; 1963.

18. Theil H. Statistical Decomposition Analysis. Amsterdam/London: North-Holland; 1972.

19. Le Boudec JY, Thiran P, Urbanke R. Information science; Bern: 2013.

20. Mêgnigbêto E. Information flow between West African Triple Helix actors. ISSI NewsI 2014;10:14-20.

21. Mêgnigbêto E. Information flow within West African innovation systems. Triple Helix: A journal of university-industry-government innovation and entrepreneurship. [in press].

22. Leydesdorff L. Redundancy in systems which entertain a model of themselves: Interaction information and the self-organization of anticipation. Entropy 2010;12:63-79.

23. Leydesdorff L. Re: Variables independence and mutual information. Cybernetics Discussion Group CYBCOM; 2014.

24. Krippendorff K. Re: Variables independence and mutual information. Cybernetics Discussion Group CYBCOM; 2014.

How to cite this article: Mêgnigbêto E. Transmission power in some particular cases of bi- or tri-dimensional complex systems. J Sci Res 2014;3:37-45.

Source of Support: Nil, Conflict of Interest: None declared

$$
\begin{aligned}
& -\frac{p(1,0)}{p(0,0)+p(1,0)} \log _{2} \frac{p(1,0)}{p(0,0)+p(1,0)} \times(p(0,0)+p(1,0)) \\
& -\frac{p(0,1)}{p(0,1)+p(1,1)} \log _{2} \frac{p(0,1)}{p(0,1)+p(1,1)} \times(p(0,1)+p(1,1)) \\
& -\frac{p(1,1)}{p(0,1)+p(1,1)} \log _{2} \frac{p(1,1)}{p(0,1)+p(1,1)} \times(p(0,1)+p(1,1))
\end{aligned}
$$

Because $H_{X \mid Y}$ is a positive scalar, ${ }^{[9,10]}$ it is null if and only if each of the positive terms that compose it is null. Therefore,

$$
\left\{\begin{array}{l}
\frac{p(0,0)}{p(0,0)+p(1,0)} \log _{2} \frac{p(0,0)}{p(0,0)+p(1,0)} \times(p(0,0)+p(1,0))=0 \\
\frac{p(1,0)}{p(0,0)+p(1,0)} \log _{2} \frac{p(1,0)}{p(0,0)+p(1,0)} \times(p(0,0)+p(1,0))=0 \\
\frac{p(0,1)}{p(0,0)+p(1,1)} \log _{2} \frac{p(0,1)}{p(0,1)+p(1,1)} \times(p(0,1)+p(1,1))=0 \\
\frac{p(1,1)}{p(0,1)+p(1,1)} \log _{2} \frac{p(1,1)}{p(0,1)+p(1,1)} \times(p(0,1)+p(1,1))=0
\end{array}\right.
$$


The system of equation (37) becomes:

$$
\left\{\begin{array}{l}
p(0,0)=0 \text { or } p(1,0)=0 \\
p(0,1)=0 \text { or } p(1,1)=0
\end{array}\right.
$$

In system of equations (38), the connector "or" is inclusive, so the system yields $2^{3}=8$ combinations of the four probabilities $p(0,0), p(0,1), p(1,0)$ and $p(1,1)$. The same combinations are solution of $H_{Y \mid X}$ under the condition that positions are inverted in the couple in heads of column. The 7 bi-dimensional configurations that satisfy system of equations (38) are presented in Table 9. By considering each of the 8 cases for $H_{X \mid Y}=0$, $H_{Y \mid Z}=0$ and $H_{Z \mid X}=0$, we obtain $8^{3}=512$ combinations of which 12 [Table 3] satisfy each $H_{X \mid Y}=0, H_{Y \mid Z}=0$ and $H_{Z \mid X}=0$.

\section{APPENDIX 2: EQUIVALENCE BETWEEN SYSTEMS OF EQUATIONS (32) AND (33)}

Let us draw the expressions of $H_{X}, H_{Y}$ and $T_{X Y}$ from Equation 7:

$H_{X}=H_{X \mid Y}+T_{X Y}$

$H_{Y}=H_{Y \mid X}+T_{X Y}$

$T_{X}=H_{X}-H_{X \mid Y}$

Replacing Equation (41) into Equation (40) gives

$H_{Y}=H_{Y \mid X}+H_{X}-H_{X \mid Y}$
Therefore,

$$
\begin{aligned}
& H_{X \mid Y}=H_{X}-H_{Y}+H_{Y \mid X} \\
& H_{Y \mid X}=H_{Y}-H_{X}+H_{X \mid Y}
\end{aligned}
$$

Subtracting Equation (45) from Equation (44) leads to:

$H_{X \mid Y}-H_{Y \mid X}=H_{X}-H_{Y}$

Similarly,

$H_{Y \mid Z}-H_{Z \mid Y}=H_{Y}-H_{Z}$

$H_{Z \mid X}-H_{X \mid Z}=H_{Z}-H_{X}$

Summing up Equations (46) to (48) leads to:

$H_{X \mid Y}-H_{Y \mid X}+H_{Y \mid Z}-H_{Z \mid Y}+H_{Z \mid X}-H_{X \mid Z}=0$

which may also be written as follows:

$\left(H_{X \mid Y}+H_{Y \mid Z}+H_{Z \mid X}\right)-\left(H_{Y \mid X}+H_{Z \mid Y}+H_{X \mid Z}\right)=0$

According to system of equations (32), $H_{X \mid Y}=H_{Y \mid Z}=$ $H_{Z \mid X}=0$; therefore, Equation (49) is equivalent to

$H_{Y \mid X}+H_{Z \mid Y}+H_{X \mid Z}=0$

Because conditional entropies are necessary positive, Equation (50) means that each of its terms is null; therefore, $H_{X \mid Z}=0, H_{Y \mid X}=0$ and $H_{Z \mid Y}=0$ which gives system of equations (33). An analogous logical reasoning on system of equations (33) results in system of equations (32). So, the two are equivalent.

\section{Author Help: Reference checking facility}

The manuscript system (www.journalonweb.com) allows the authors to check and verify the accuracy and style of references. The tool checks the references with PubMed as per a predefined style. Authors are encouraged to use this facility, before submitting articles to the journal.

- The style as well as bibliographic elements should be $100 \%$ accurate, to help get the references verified from the system. Even a single spelling error or addition of issue number/month of publication will lead to an error when verifying the reference.

- $\quad$ Example of a correct style

Sheahan P, O'leary G, Lee G, Fitzgibbon J. Cystic cervical metastases: Incidence and diagnosis using fine needle aspiration biopsy. Otolaryngol Head Neck Surg 2002;127:294-8.

- Only the references from journals indexed in PubMed will be checked.

- Enter each reference in new line, without a serial number.

- $\quad$ Add up to a maximum of 15 references at a time.

- If the reference is correct for its bibliographic elements and punctuations, it will be shown as CORRECT and a link to the correct article in PubMed will be given.

- If any of the bibliographic elements are missing, incorrect or extra (such as issue number), it will be shown as INCORRECT and link to possible articles in PubMed will be given. 